\title{
Impact of COVID-19 on adolescents' mental health: a systematic review
}

\author{
Gilbert Sterling Octavius ${ }^{1 *}$ (D, Felicia Rusdi Silviani ${ }^{1}$, Alicya Lesmandjaja', Angelina ${ }^{2+}$ and Andry Juliansen ${ }^{2+}$
}

\begin{abstract}
Background: The impact of COVID-19 towards psychology and mental health is anticipated to be significant and may affect the population disproportionately, especially adolescent as the vulnerable category. We aimed to analyze the impact of COVID-19 towards adolescents' mental health.

Main body: A systematic search was conducted from Cochrane, Google Scholar, Scielo, and PubMed. Inclusion criteria included all types of studies which observed the effect of COVID-19 and its related causes, such as lockdown, on adolescents' mental health. All studies were assessed for its level of evidence according to Oxford 2011 criteria and Newcastle Ottawa Scale (NOS). Three studies (Seçer and Ulaş, Int J Ment Health Addict: 1-14, 2020; Zhou et al., Eur Child Adolesc Psychiatry 29:749-58, 2020; Qu et al., Lancet: 1-17, 2020) showed that COVID-19 was a risk factor for mental health problems in adolescents while Oosterhoff et al. (J Adolesc Health 67: 179-185, 2020) showed that adolescents who preferred to stay at home during this pandemic reported less anxiety and depressive symptoms
\end{abstract}

Conclusion: COVID-19 has been found to be associated with mental health changes in adolescents which meant management of COVID-19 should also focus on mental health as well.

Keywords: COVID-19, Mental health, Adolescent, Depression, Anxiety

\section{Background}

Coronavirus disease-19 (COVID-19) was found initially in Wuhan, Hubei Province, China, on December 31, 2019, and it continues to be a pandemic [1]. Until July 13,2020 , it has infected about $12,750,275$ people and caused 566,355 deaths around the world while showing no signs of slowing down [2]. Based on this fact, many countries around the world had applied physical distancing and closed public places such as schools, campuses, offices, and public places to curb the transmission [3-6]. On the other hand, physical distancing has impacted mental health by depriving social contact, especially the adolescent $[1,7,8]$. Adolescent is defined as individuals in the 10-19 years age group [9] in which it is a

\footnotetext{
* Correspondence: Sterlinggilbert613@hotmail.com

${ }^{+}$Angelina and Andry Juliansen contributed equally to this work.

${ }^{1}$ Faculty of Medicine, University of Pelita Harapan, Karawaci, Tangerang,

Banten, Indonesia

Full list of author information is available at the end of the article
}

vulnerable age group to develop negative mental health impairment because they are very sensitive to psychological and social transformation. Adolescent experiences higher peer interaction and social world than with their family, and even forms complex peer relationship compared to their younger counterparts such as babies and children. Any separation from peer relationships such as rejection, bullying, or loneliness has been linked to mental health disorder such as depression, anger, fear, stress, and anxiety [10]. Physical distancing had led individuals to cut off social interaction unintentionally because individuals had the tendencies to avoid conversation in order to limit meetings. Although most adolescents were exposed to physical distancing, lockdown, or quarantine during this pandemic of COVID-19, the adolescents' mental health changes can be variative based on their circumstances and motivation to obey physical distancing [11]. This study aims to analyze the impact of COVID-19 towards adolescent's mental health, with the hypothesis

\section{SpringerOpen}

(c) The Author(s). 2020 Open Access This article is licensed under a Creative Commons Attribution 4.0 International License, which permits use, sharing, adaptation, distribution and reproduction in any medium or format, as long as you give appropriate credit to the original author(s) and the source, provide a link to the Creative Commons licence, and indicate if changes were made. The images or other third party material in this article are included in the article's Creative Commons licence, unless indicated otherwise in a credit line to the material. If material is not included in the article's Creative Commons licence and your intended use is not permitted by statutory regulation or exceeds the permitted use, you will need to obtain permission directly from the copyright holder. To view a copy of this licence, visit http://creativecommons.org/licenses/by/4.0/. 
that COVID-19 is associated with poor mental health outcomes on adolescents.

\section{Methods}

This systematic review was conducted based on the Preferred Reporting Items for Systematic Review and MetaAnalysis (PRISMA) statement [12]. The protocol of this systematic review has been registered in The International Prospective Register of Systematic Reviews (PROSPERO) database (CRD42020195764).

We included cohort studies and cross-sectional studies which observed the effect of COVID-19 and its related causes such as lockdown on adolescents' mental health impact such as depression, fear, and anger. Exclusion criteria comprise of studies that include correspondents with age group extending below and over the adolescents' age range (10-19 years old), as well as studies which include adolescents with pre-existing mental health problems such as autism spectrum disorder (ASD) or post-traumatic stress disorder (PTSD).

A systematic literature search was performed on July 7, 2020, using four different databases such as Cochrane, Google Scholar, Scielo, and PubMed using keywords listed in Table 1. Literature selection was performed from 2019 onwards and restricted to only publications that are in English language.

Data from each study was extracted in a standardized form, compiling study citations, baseline characteristics of the included subjects, and the study findings. Study citations included the name of the first author, year of publication, and title of the study. Meanwhile, characteristics of each study refered to study design, location of the study, and patients' characteristics (age, ethnicity, gender, sample size, and family income). The study findings extracted involved the odds ratio, $R^{2}$, or $\beta$ value analyzed in each study.

Three independent reviewers conducted the quality assessment of the studies (GS, FR, AL). The included studies were critically appraised using Newcastle Ottawa Quality Assessment Scale (NOS) for case control and cohort studies [13]. Any discrepancies of NOS score between reviewers were discussed until it reached a conclusion. If the discrepancies are still not settled, two expert reviewers (A, AJ) were consulted, and decisions were made by them. High-quality studies were defined as studies fulfilling NOS score of minimum 7 (Table 2). Data was synthesized based on a minimum of four different and high-quality studies with consistent finding. The obtained data was analyzed considering the method of variable analysis used, study size, odds/hazard ratio, along with its confidence interval.

\section{Results}

Literature search was done using keywords listed in Table 1. Out of 5876 articles identified, 48 articles were retrieved after title and abstract screening. Duplicates were then removed, and after ensuring the remaining articles were appropriate according to the writers' inclusion and exclusion criteria, four articles were chosen for this study which were studies by $\mathrm{Qu}$ et al. [14], Oosterhoff et al. [15], Zhou et al. [11], and Seçer and Ulaş [16]. The flow of our study selection is presented in Fig. 1 according to the PRISMA statement [12].

The summary of baseline study characteristics is presented in Table 3. All studies had level 2 of evidence based on Oxford 2011 and were considered high-quality based on each NOS score (Table 2). All studies were cross-sectional studies except for Qu et al. [14], which was a prospective study, which means that temporality or causality cannot be established. Each study also used different questionanires to assess pyschosocial impact and mental health except Zhou et al. [11] and Qu et al. [14] which both used Patient Health Questionnaire (PHQ-9) and Generalized Anxiety Disorder Scale (GAD7) to assess depression and anxiety, respectively. All of the questionnaires are self-administered online except in the study of Qu et al. [14] which initially was administered by trained staffs.

The study done by Zhou et al. [11] showed that prevalence of mild-to-severe depressive and anxiety symptoms in Chinese adolescents during COVID-19 outbreak was $43.7 \%$ and $37.4 \%$ respectively. The prevalence of adolescents with both depressive and anxiety symptoms was $31.3 \%$. They found that adolescents living in cities were

Table 1 Keywords used in each search engine

\begin{tabular}{|c|c|}
\hline Search engine & Keywords \\
\hline PubMed & $\begin{array}{l}\text { (((()(((“"COVID-19"[Title] OR "COVID-2019"[Title]) OR "severe acute respiratory syndrome coronavirus 2"[Title]) OR "2019-nCoV"[Title]) } \\
\text { OR "SARS-CoV2"[Title]) OR "2019nCoV"[Title]) OR "Wuhan"[Title]) OR "coronavirus"[Title]) AND "Adolescent"[Title]) AND ("psychological } \\
\text { impact"[Title] OR "mental health"[Title]) }\end{array}$ \\
\hline Scielo & $\begin{array}{l}\text { ((((ti:(covid-19))) OR ((ti:(covid-2019))) OR ((ti:(severe acute respiratory syndrome coronavirus 2))) OR ((ti:(2019-ncov))) OR } \\
\text { ((ti:(sars-cov2))) OR ((ti:(wuhan))) OR ((ti:(2019ncov)))) OR (ti:(coronavirus))) AND ((ti:(adolescent))) AND ((ti:(psychological impact)) OR } \\
\text { (ti:(mental health))) }\end{array}$ \\
\hline Cochrane & $\begin{array}{l}\text { (covid-19 OR covid-2019 OR severe acute respiratory syndrome coronavirus } 2 \text { OR 2019-ncov OR sars-cov2 OR wuhan OR 2019ncov } \\
\text { OR coronavirus) AND (adolescent) AND (psychological impact OR mental health) }\end{array}$ \\
\hline Google Scholar & COVID-19 AND Psychological Impact AND Adolescent AND Mental Health \\
\hline
\end{tabular}


Table 2 Quality assesment of the included studies using Newcastle Ottawa Scale

\begin{tabular}{lllll}
\hline Type of study & Selection & Comparability & Outcome & Total \\
\hline Oosterhoff et al. [15] & $* * * *$ & $*$ & $* *$ & 7 \\
Seçer and Ulaş [16] & $* * * *$ & $*$ & $* *$ & 7 \\
Zhou et al. [11] & $* * * *$ & $*$ & $* * *$ & 8 \\
Qu et al. [14] & $* * * *$ & $*$ & $* *$ & 7
\end{tabular}

Each * represents a point for fulfilling the NOS criteria

less depressed (37.7\%). Zhou et al. [11] also found that adolescents living in cities were less likely to have depressive or anxiety symptoms $(37.7 \%$ vs $47.5 \%$ and $32.5 \%$ vs 40.4\%). The scores in COVID-19 knowledge, prevention, and control measures, and projections of COVID-19 were higher in adolescents without depressive and or anxiety symptoms. Utilizing multivariate logistic regression analysis, Zhou et al. [11] found that female gender $\left(\mathrm{OR}_{(\text {Depres- }}\right.$ $\operatorname{sion}[\mathrm{DE}])=1.15,1.05-1.26 ; \mathrm{OR}_{(\text {Anxiety[AN] })}=1.10,1.00-$ 1.21), living in Hubei province $\left(\mathrm{OR}_{\mathrm{DE}}=1.58,1.34-1.87\right.$; $\left.\mathrm{OR}_{\mathrm{AN}}=1.64,1.39-1.93\right)$, and being in the junior grade three $\left(\mathrm{OR}_{\mathrm{DE}}=1.40,1.11-1.75 ; \mathrm{OR}_{\mathrm{AN}}=1.32,1.04-1.67\right)$ were risk factors for depressive and anxiety symptoms.

The study done by Qu et al. [14] is the only study included to be using prospective cohort as its study design.
First, the authors did the first survey round as a baseline data which assessed their background factors, depression, anxiety, resilience, and childhood maltreatment using the questionnaires listed in Table 3 . The second round of survey was done 3 months after the first round of data gathering, and after excluding invalid questionnaires the valid follow-up rate was $74.91 \%$ and varied by region $(p<0.001)$. They found that just before the pandemic, $51.51 \%$ of adolescents reported depressive symptoms and $38.53 \%$ reported anxiety symptoms. After home confinement, the number dropped to $38.29 \%$ and $23.73 \%$ respectively (all $p<0.0001$ ). After Propensity Score Matching (PSM) matching, adolescents with exposure risk still had more depression $(60.54 \% ; p=$ $0.0023)$ and anxiety symptoms $(41.26 \% ; p=0.0072)$ than those without any exposure risks $(45.95 \%$ and $28.83 \%$ respectively). Exposure risk was defined as anyone in the surrounding living environment of the participant who was infected with COVID-19. Qu et al. concluded that adolescents with exposure risks (assessed with assessed with PSM analysis [PSM] analysis) are 2.3 times more likely to suffer from depression (1.7$3.1 ; p<0.0001)$ and 2.1 times more likely to suffer from anxiety symptoms $(1.6-2.8 ; p<0.0001)$ in adolescents with exposure risk.
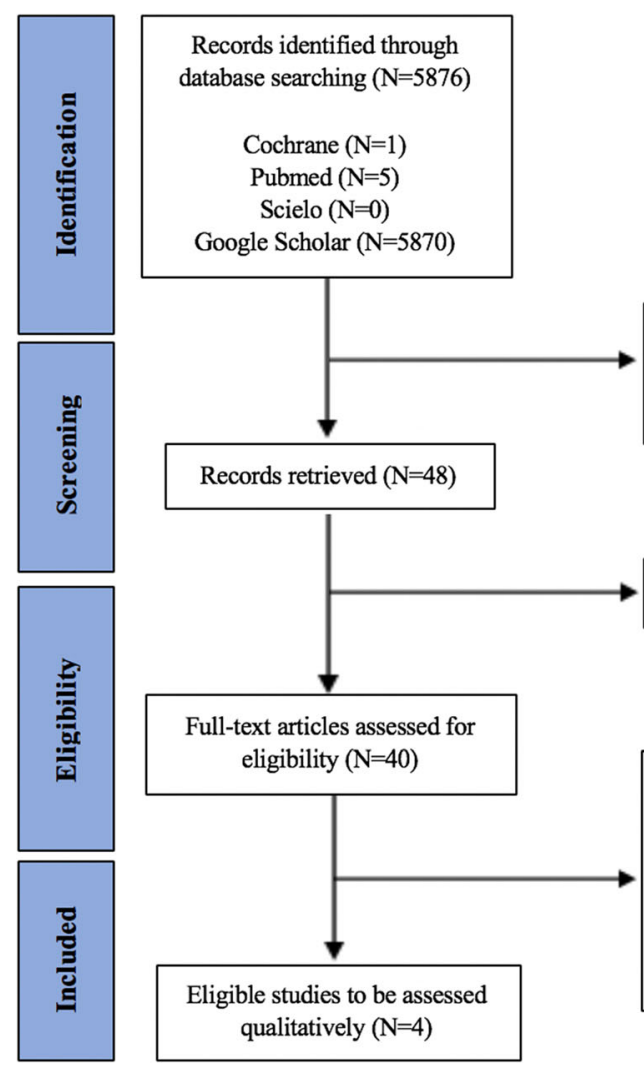

Records exluded based on eligibility criteria after title and abstract screening $(\mathrm{N}=5828)$

Duplicates excluded $(\mathrm{N}=8)$

Full-text article excluded ( $\mathrm{N}=35)$ due to:

Underlying psychiatric disease $(\mathrm{N}=1)$

Age range included children and/or adult $(\mathrm{N}=16)$

Article not written in English language ( $\mathrm{N}=1)$ Editorial paper $(\mathrm{N}=12)$

Article talking about impact of lockdown in other pandemic $(\mathrm{N}=6)$

Fig. 1 PRISMA flowchart of study selection 


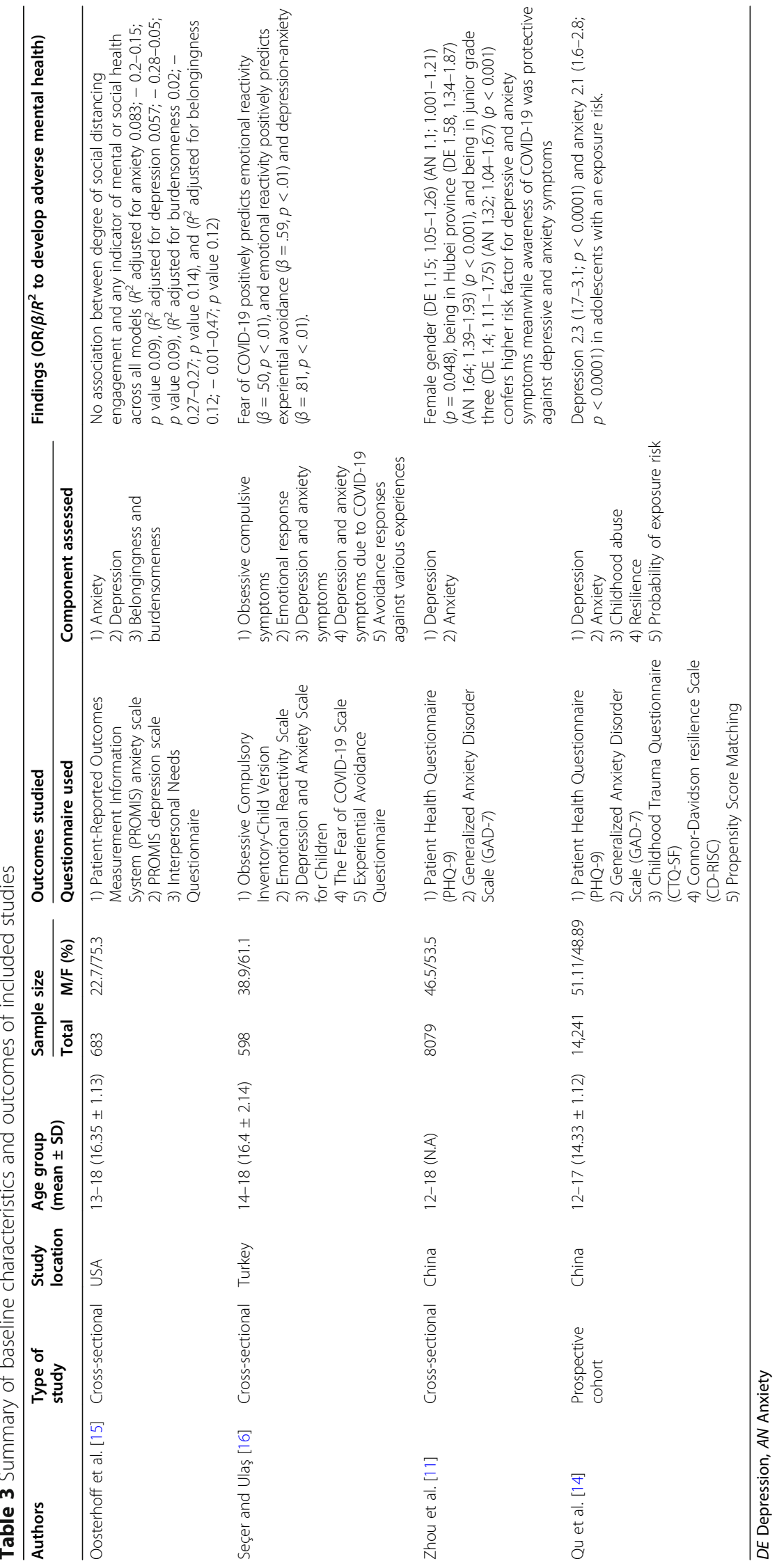


Using standardized structural equation modeling (SEM), Seçer and Ulaş [16] found that fear of COVID-19 positively predicts emotional reactivity $(\beta=.50, p<0.01)$ which positively predicts experiential avoidance $(\beta=.59$, $p<0.01)$ and depression-anxiety $(\beta=.81, p<0.01)$. Oosterhoff et al. [15] did not find any association between degree of social distancing and any indicator of mental or social health. This study also found that those who were social distancing because of social responsibility reported less anxiety symptoms $\left(R^{2}=0.03\right.$, standard error $(\mathrm{SE})=0.12[-0.21,0.27])$, depressive symptoms $\left(R^{2}=-0.05, \mathrm{SE}=0.12[-0.29,0.19]\right)$, and burdensomeness $\left(R^{2}=-0.35, \mathrm{SE}=0.19[-0.73,0.03]\right)(p<0.05)$ which was also found in adolescents who would have preferred to stay at home $\left(R^{2}\right.$ anxiety $=-0.3, \mathrm{SE}=0.11[-$ $0.51,-0.09], R_{\text {depressive }}^{2}=-0.35, \mathrm{SE}=0.1[-0.55,-$ $0.14]$, and $R_{\text {belongingness }}^{2}=-0.03, \mathrm{SE}=0.16[-0.29,0.35)$ $(p<0.05)$. Additionally, adolescents who were social distancing because a friend told them they should reported greater depressive symptoms $\left(R^{2}=0.26, \mathrm{SE}=0.13[0.02\right.$, $0.51])(p<0.05)$ while adolescents who were social distancing because they wanted to avoid judgment reported greater anxiety symptoms $\left(R^{2}=0.35, \mathrm{SE}=0.17[0.03\right.$, $0.68])(p<0.05)$.

\section{Discussion}

COVID-19 is an unprecedented infectious disease which has taken a massive toll on deaths. Sudden changes in lifestyle, including lockdown, quarantine, or physical distancing coupled with the loss of loved ones, may take a toll on mental and emotional health. Although there has been several studies in the past trying to elucidate how quarantine and isolation affect mental health, the extent on how far adolescents' mental health is affected is unknown [17]. In this systematic review, three included studies show that COVID-19 impacts on adolescents' mental health with one study citing that there was no significant impact.

The cohort result from Qu et al. [14] should be interpreted carefully. There was $25.09 \%$ drop-out from the participants in the first round to the second round, so the result from first and second round might not be able to be compared directly. The study also found that the incidence and severity of anxiety and depressive symptoms dropped significantly after home confinement. It meant that mental health was not affected in overall adolescent population, but only in exposure risk population. Furthermore, there was only $2.2 \%$ subjects that reported a risk of exposure. As this study analyzed COVID-19 exposure risk as independent variable, the low number of subjects in exposure risk might not be able to represent a real general population. Despite its limitations, this study had a baseline data to compare pre-home confinement and intra-home confinement, which made it one of the best quality studies among the others.

Zhou et al. [11] presented a similar finding with $\mathrm{Qu}$ et al. [14] that living in community with high number of COVID-19 cases was a risk factor towards depression and anxiety. These two studies also used same questionnaires to assess depressive and anxiety symptoms. However, Zhou et al. [11] found that awareness of COVID19 was a protective factor against depressive and anxiety symptoms while Qu et al. [14] stated that some protective factors, such as good parent-child relationship, few adverse experiences, good family structure, and high resilience could be outweighed by exposure of COVID-19. These different results showed that there were a lot of factors that might affect the rise of depressive and anxiety symptoms in adolescent in COVID-19 pandemic era.

Study by Seçer and Ulaş was unique because it is the only study which assessed obsessive-compulsive disorder (OCD) due to fear of COVID-19 [16]. The reasoning behind it was due to the fear of this disease, adolescents will start a washing and hoarding obsession. Although the study did not mention the number of positive cases in the area, the common denominator of fear of COVID-19 is still maintained in this study. Seçer and Ulaş [16] went a step further by theorizing that emotional reactivity might be one of the various psychopathologies that might explain depression, anxiety, and OCD in adolescents while experiential avoidance mediates fear of COVID-19 and OCD symptoms. However, while trying to establish a causality, this paper is a crosssectional study which means that further studies need to consider other study designs in order to establish the direct link between fear of COVID-19 with OCD symptoms, if possible.

Although the study done by Oosterhoff et al. [15] found that there was no association between degree of social distancing and mental or social health, this study found that motivations for social distancing were differentially associated with degree of social distancing as well as depressive symptoms, anxiety symptoms, burdensomeness, and belongingness. Adolescents who were social distancing because they wanted to avoid social judgment reported more anxiety symptoms which might be explained by the fact that past researches shows that symptomatic youths are more sensitive towards social judgment or peer rejection [18]. This study also found that adolescents who prefer to stay at home reported lower anxiety and depressive symptoms because adolescents who chose to stay at home might be struggling less with reduced social contact [15]. However, some motivations studied have low samples such as "no alternatives" (17.8\%), "friends said I should" (13.9\%), "avoid judgment" (7\%), and others (4.4\%) which could affect the 
significancy of the associations studied. High compliance towards social distancing in the sample population could be seen from the motivations of social responsibility (78.1\%) and not wanting others to get sick (77.9\%). It implies that the adolescents in the population studied have a good knowledge of COVID-19 which confers protective factor towards depression and anxiety just like in the study done by Zhou et al. [11].

Onset of depression is affected by both genetic factors and external environmental factors such as stressful life events $[19,20]$. Adolescents are especially vulnerable to stressful life events and could lead to lower levels of motivation, lower concentration, poorer achivement [21], psychological distress, anxiety, depression [22], and suicide [23]. In fact, mental health conditions account for $16 \%$ of the global burden of disease and injury in people aged 10-19 years old and half of all mental health conditions starting at 14 years old, but most cases were undetected and untreated [24]. Moreover, non-emergency medical services were halted or redirected towards more emergency cases and hence medical care for affected adolescents will be affected [25]. Fear of COVID-19 significantly increased negative affect, anxiety, and depression [26]. Qu et al. [14] stated that long-term confinement had no adverse mental health impact on adolescents from regions with a low incidence of COVID-19. Compared to Qu et al. [14], the exposure risk of COVID-19, living area, and fear of COVID-19 which could be the risk factors for mental health changes on adolescents are not measured in Oosterhoff et al.'s study [15]. A study done by Fitzpatrick stated that the fear of COVID-19 is not distributed uniformly across the USA, and there were specific concentrated COVID-19 fear in more crowded populated communities, communities with higher presumptive and reported cases of COVID-19, and urban locations [27]. Most studies were also originated from high-income countries, which may affect the generalizability of the findings to low-income and middle-income countries which are often under-presented in terms of generating evidences through empirical studies [28]. Therefore, the result which was conducted in high income countries such as the Oosterhoff's [15] study cannot be generalized to other low- and middle-income countries that were exposed to COVID-19.

Adolescents who are infected by COVID-19 would also need psychiatric care as $10 \%$ of infected children who experienced trauma due to infection and its consequences might be diagnosed with PTSD [29]. Psychiatrists and pediatricians need to be aware of warning signs of mental health problems in COVID-19 infected children such as mood swings and psychosis-like symptoms as psychiatrists need to work directly with young patients and their families in order to screen and detect mental disorders as soon as possible with adequate personal protective equipment [25].
Zhou et al.'s [11] study suggested that the level of knowledge, prevention, and control measures for COVID-19 are protective factors against the development of depressive and anxiety symptoms. However, there are media/press sources that might give false information and reports about COVID-19 which could lead to anxiety and depressive symptoms of the public. A study by Zhong et al. also suggested that health education aimed at increasing knowledge of COVID-19 are important to keep optimistic attitudes [30]. This is related to a statement from Zhou's study that said positive and optimistic attitudes towards COVID-19 epidemic's development are also protective factors against depressive and anxiety symptoms [11].

The government and health authorities' role are important in COVID-19 crisis to provide adequate information and deny any false information to keep the public informed. In crisis situation like this, it is convenient to educate and provide public with the accurate information through digital media platform. A study by Liu suggested that the uses of digital media might initiate preventive behaviors directly or indirectly. It states that seeking information about COVID-19 from online news media, media social networking (MSNs), social live streaming services (SLSSs) was associated with increased preventive practices [31]. Since there are a lot of misinterpreted informations from the internet, it is important to obtain information from a credible website such as the national Centers for Disease Control (CDC), World Health Organization (WHO), or from other sources endorsed by these authorities, rather than a general search on the internet or social media [32].

A rapid systematic review done by Imran et al. found that quarantine is associated with significant negative impact on mental health of children and adolescents which might persist for months or years after the quarantine. Although the cause of quarantine is very diverse (from natural disasters such as Tsunami in Aceh 2004 to children requiring ventilators at home), this study proposed some interventions that could be done to reduce the impact of mental health during quarantine such as provision of psychosocial support, dissemination of accurate information, limit exposure to news, positive parenting, social connectivity, as well as behavior activation complemented with sleep hygiene, exercise, and healthy eating [33]. Another systematic review done by Loades et al. [34] which assessed the impact of social isolation and loneliness due to COVID-19 on children's and adolescent's mental health found that children and adolescents are more prone towards high rates of depression and anxiety during and after enforced isolation ends which might last up to 0.25 to 9 years later.

Our study is limited by exclusion of articles written in languages other than English as well as unexplored gray 
literatures. The studies included also did not measure any baseline characteristics except for study done by $\mathrm{Qu}$ et al. [14]. Moreover, this systematic review could not be proceeded to meta-analysis due to the heterogenous use of variable analysis. Therefore, we recommend future studies to use a more homogenous questionnaire as well as their odds ratio presentation clearly to depict the effect of COVID-19's mental health impact in adolescents. Lastly, correlation between those who were at low risk of contracting COVID-19 and the lesser impact on mental health risk may be independent of the actual risk and was just based on individual's subjective perceptions.

Taken together, this is the first systematic review that shows the impact of COVID-19 on mental health among adolescents. Hence, this study might be important in the aspect of a more holistic approach towards adolescents who are affected by COVID-19, whether directly or indirectly. In the long term, there might be a surging incidence and prevalence of mental health disorders which might be attributed to COVID-19 and hence awareness and interventions are needed which might require cooperations between the adolescents, families, medical care workers, and the governments.

\section{Conclusion}

COVID-19 has been found to be associated with adolescents mental health changes, especially the fear of COVID-19 in a population with adequate exposure of COVID-19 was proved to create adverse mental health condition such as anxiety and depression. adolescents who had experienced previous trauma with addition of social isolation/quarantine and loneliness were more prone towards anxiety and depression during and even after the enforced isolation ends. On the other hand, some protective factors were found to help adolescents stay away from any mental health adverse impacts due to COVID-19. Physical-psychosocial support provision, adequate and accurate information from credible source about COVID-19, and good motivation to obey physical distancing has shown to decrease the likelihood of negative mental health changes in adolescent. Through this systematic review, psychiatrist, pediatrician, parents, or other parties who accompany or take care of adolescents hopefully can raise awareness to detect mental health changes in order to decrease adverse mental health impacts in adolescent's future. Further study needs to be done to find other factors which may be associated to mental health changes besides fear of COVID-19 and social distancing.

\section{Abbreviations}

ASD: Autism spectrum disorder; CDC: Centers for Disease Control; COVID-

19: Coronavirus disease-19; GAD: Generalized Anxiety Disorder Scale; MSN: Media Social Networking; NOS: Newcastle Ottawa Scale;

OCD: Obsessive compulsive disorder; PHQ: Patient Health Questionnaire; PRIS
MA: Preferred Reporting Items for Systematic Reiew and Meta-Analysis; PROSPERO: The International Prospective Register of Systematic Reviews; PSM: Propensity Score Matching; PTSD: Post-traumatic stress disorder; SLSS: Social Live Streaming Services; WHO: World Health Organization

\section{Acknowledgements}

Not applicable

Authors' contributions

GSO, FRS, and AL did the conception of this systematic review while GSO registered the procedure on PROSPERO website. Data collections are done by GSO, FRS, and AL with disputes between the articles that were selected and settled by A and AJ. GSO and FRS drafted the article while A and AJ did critical revision of the article. Final approval of the version to be published was granted by all authors.

\section{Funding}

The authors declare that the research was conducted in the absence of any financial grants.

\section{Availability of data and materials}

Available upon request

\section{Competing interest}

The authors declare no conflict of interest.

Ethics approval and consent to participate Not applicable

\section{Consent for publication}

All authors have read and agreed on publishing this article

\section{Author details}

${ }^{1}$ Faculty of Medicine, University of Pelita Harapan, Karawaci, Tangerang, Banten, Indonesia. '2Department of Pediatric, Faculty of Medicine, University of Pelita Harapan, Karawaci, Tangerang, Banten, Indonesia.

Received: 16 October 2020 Accepted: 22 November 2020

Published online: 22 December 2020

\section{References}

1. Wang C, Pan R, Xi W, Tan Y, Xu L, Ho CS et al (2020) Immediate psychological responses and associated factors during the initial stage of the 2019 coronavirus disease (COVID-19) epidemic among the general population in China. Int J Environ Res Public Health 17:1729

2. World Health Organization (2020) WHO coronavirus disease (COVID-19) dashborad Available from: https://covid19.who.int/

3. Bhat BA, Khan S, Manzoor S, Niyaz A, Tak HJ, Anees S et al (2020) A study on impact of COVID-19 lockdown on psychological health, economy and social life of people in Kashmir. International Journal of Science and Healthcare Research.5(2):36-46

4. de Figueiredo AM, Codina AD, Figueiredo DCMM, Saez M, León AC (2020) Impact of lockdown on COVID-19 incidence and mortality in China : an interrupted time series study. Bull World Health Organ:1-19

5. Ahmad A, Nourah P, Abdulrahman B (2020) Factors influencing mental health during Covid-19 outbreak: an exploratory survey among Indian population, pp 1-16

6. Veer IM, Riepenhausen A, Zerban M, Wackerhagen C, Hajduk M Mental resilience in the Corona lockdown: first empirical insights from Europe. Psyarxiv Prepr, pp 1-15

7. Jiao WY, Wang LN, Liu J, Fang SF, Jiao FY, Pettoello-Mantovani M et al (2020) Behavioral and emotional disorders in children during the COVID-19 epidemic. Eur Pediatr Assoc 221:264-266

8. Holmes EA, Connor RCO, Perry VH, Tracey I, Wessely S, Arseneault L et al (2020) Position paper multidisciplinary research priorities for the COVID-19 pandemic: a call for action for mental health science, pp 547-560

9. Sawyer SM, Azzopardi PS, Wickremarathne D, Patton GC (2018) The age of adolescence. The Lancet Child \& Adolescent Health. 2(3):223-8. https://doi. org/10.1016/S2352-4642(18)30022-1

10. Orben A, Tomova L, Blakemore S (2020) Viewpoint the effects of social deprivation on adolescent development and mental health. Lancet child 
Adolesc Heal 4642:1-7Available from:. https://doi.org/10.1016/\$23524642(20)30186-3

11. Jiang S, Li Z, Zhang G, Lei L, Zhao W, Guo C et al (2020) Prevalence and socio-demographic correlates of psychological health problems in Chinese adolescents during the outbreak of COVID-19. Eur Child Adolesc Psychiatry 29:749-758Available from. https://doi.org/10.1007/s00787-020-01541-4

12. Moher D, Shamseer L, Clarke M, Ghersi D, Liberati A, Petticrew M et al (2015) Preferred reporting items for systematic review and meta-analysis protocols (PRISMA-P) 2015 statement, pp 1-9

13. GA Wells, B Shea, D O'Connell, J Peterson, V Welch, M Losos PT. The Newcastle-Ottawa Scale (NOS) for assessing the quality of nonrandomised studies in meta-analyses. Available from: http://www.ohri.ca/programs/ clinical_epidemiology/oxford.asp. Accessed 7 Jul 2020.

14. Qu M, Yang K, Cao Y, Si T, Wang X, Xiu MH et al (2020) Mental health status of adolescents after family confinement during the COVID-19 outbreak. Lancet::1-17

15. Oosterhoff B, Palmer CA, Wilson J, Shook N (2020) Adolescents' motivations to engage in social distancing during the COVID-19 pandemic: associations with mental and social health. J Adolesc Health; 67(2): 179-85. Available from https://doi.org/10.1016/j.jadohealth.2020.05.004.

16. Seçer I, Ulaş S (2020) An investigation of the effect of COVID-19 on OCD in youth in the context of emotional reactivity, experiential avoidance, depression and anxiety. Int J Ment Heal Addicition:1-14. https://doi.org/10. 1007/s11469-020-00322-z [Epub ahead of print]

17. Imran N, Zeshan M, Pervaiz Z (2020) Mental health considerations for children \& adolescents in COVID-19 pandemic. Pak J Med Sci. 36:67-72

18. Platt B, Kadosh KC, Lau JYF (2013) The role of peer rejection in adolescent. Depress Anxiety 821:809-821

19. Kendler KS, Karkowski LM, Ph D, Prescott CA, Ph D (1999) Causal relationship between stressful life events and the onset of major depression. Am J Psychiatry; 156(6):837-841. https://doi.org/10.1176/ajp.156.6.837.

20. Uchida S, Hara K, Kobayashi A, Otsuki K, Yamagata H, Hobara T (2011) Article epigenetic status of Gdnf in the ventral striatum determines susceptibility and adaptation to daily stressful events. Neuron 69:359-372Available from: https://doi.org/10.1016/..neuron.2010.12.023

21. Compas BE (1987) Coping with stress during childhood and adolescence. Am Psychol Assoc 1:393-403

22. Hassanzadeh A, Heidari Z, Feizi A, Keshteli AH, Roohafza $H$, Afshar $H$ et al (2017) Association of stressful life events with psychological problems: a large-scale community-based study using grouped outcomes latent factor regression with latent predictors.

23. World Health Organization (2019) Adolescent mental health Available from: https:/www.who.int/news-room/fact-sheets/detail/adolescent-mentalhealth

24. Kessler RC, Angermeyer M, Anthony JC, De Graaf R, Demyttenaere K, Gasquet I et al (2007) Lifetime prevalence and age-of-onset distributions of mental disorders in the World Health Organization's. World Psychiatry 6: 168-176

25. Cui Y, Li Y, Zheng Y, Society C, Psychiatry A (2020) Mental health services for children in China during the COVID-19 pandemic: results of an expert-based national survey among child and adolescent psychiatric hospitals. Eur Child Adolesc Psychiatry:5-10Available from. https://doi.org/10.1007/s00787-02001548-x

26. Li HY, Cao H, Leung DYP (2020) The psychological impacts of a COVID-19 outbreak on college students in China : a longitudinal study

27. Fitzpatrick KM, Harris C, Drawve G (2020) Fear of COVID-19 and the mental health consequences in. America. 12:17-21

28. Hossain M, Sultana A, Purohit N (2019) Mental health outcomes of quarantine and isolation for infection prevention: a systematic umbrella review of the global evidence, pp 1-11

29. Gutermann J, Schreiber F, Matulis S, Schwartzkopff L (2016) Psychological treatments for symptoms of posttraumatic stress disorder in children, adolescents, and young adults. Clin Child Fam Psychol Rev. 77-93. Available from: https://doi.org/10.1007/s10567-016-0202-5.

30. Zhong B, Luo W, Li H, Zhang Q, Liu X, Li W et al (2020) Knowledge, attitudes, and practices towards COVID-19 among Chinese residents during the rapid rise period of the COVID-19 outbreak: a quick online crosssectional survey. Int J Biol Sci 16:1745-1752

31. Liu PL (2020) COVID-19 information seeking on digital media and preventive behaviors. Cyberpsychology, Behavior, and Social Networking 23(10):1-6. https://doi.org/10.1089/cyber.2020.0250
32. Li W, Liao J, Li Q, Baskota M, Wang X, Tang Y et al (2020) Public health education for parents during the outbreak of COVID-19: a rapid review. Ann Transl Med 8:1-11

33. Imran N, Aamer I, Sharif Ml, Bodla ZH, Naveed S (2020) psychological burden of quarantine in children and adolescents: a rapid systematic review and proposed solutions. Pak J Med Sci 36(5):1 106-16

34. Loades ME, Chatburn E, Higson-sweeney N, Reynolds S, Shafran R, Brigden A et al (2020Available from) Rapid systematic review: the impact of social isolation and loneliness on the mental health of children and adolescents in the context of COVID-19. J Am Acad Child Adolescent Psych. https://doi. org/10.1016/j.jaac.2020.05.009

\section{Publisher's Note}

Springer Nature remains neutral with regard to jurisdictional claims in published maps and institutional affiliations.

\section{Submit your manuscript to a SpringerOpen ${ }^{\circ}$ journal and benefit from:}

- Convenient online submission

- Rigorous peer review

- Open access: articles freely available online

High visibility within the field

- Retaining the copyright to your article

Submit your next manuscript at $\boldsymbol{\nabla}$ springeropen.com 